ANDREAS DORN

\title{
Zur Lehre Amunnachts: Ein Join und Missing Links*
}

\section{Einleitung}

Der Weisheitstext wurde durch Amunnacht, Sohn des Ipui, verfasst. Amunnacht wurde in der ausgehenden 19. Dynastie geboren und war während der ersten Hälfte der 20. Dynastie vom 16. Regierungsjahr Ramses' III. (ca. 1167 v. Chr.) bis maximal im 7. Regierungsjahr Ramses' VI. (ca. 1134 v. Chr.) Oberschreiber der königlichen Grabbauverwaltung in ThebenWest. Zusammen mit der Lehre Anis stellen diese beiden in Neuägyptisch abgefassten Weisheitstexte bis zu Beginn des 1. Jahrtausends v. Chr. die einzigen Vertreter der Gattung dar, bei deren Verfassern es sich nicht um Pseudoepigraphen handelt ${ }^{2}$. Diese Tatsache sowie auch das Vorliegen zahlreicher weiterer Texte verschiedenster Gattungen, die Amunnacht geschrieben und signiert hat ${ }^{3}$, führten in jüngster

* Der Aufsatz entstand im Rahmen des Projekts „Mikrogeschichte und Archäologie: Die Biografie des ägyptischen Schreibers Amunnacht (ca. *1190-†1140 v. Chr.)“, das durch ein Postdoc-Stipendium der Universität Basel sowie unter dem Titel „La Biographie du scribe égyptien Amennakhte (environ *1190-†1140 av. j.-c.)" durch eines der Université de Liège gefördert wurde. Für Anregungen und Kommentare danke ich S. Polis.

${ }^{1}$ Nach wie vor grundlegend $\mathrm{zu}$ seinem Leben, zu zahlreichen von ihm verfassten Texten und zu seiner Laufbahn ist die Darstellung bei J. Černý, Community of Workmen at Thebes in the Ramesside Period (BdE 50), Le Caire 1973, 339-352.

${ }^{2}$ Zum Verfasser der Lehre des Ani siehe die Angaben bei J. F. Quack, Die Lehren des Ani. Ein neuägyptischer Weisheitstext in seinem kulturellen Umfeld (OBO 141), Freiburg/Göttingen 1994, 79-81. Abgesehen von den Angaben zu Ani, die der Text selbst bereitstellt, sind bisher keine Zeugnisse mit dem Verfasser „Ani“ in Verbindung gebracht worden, so dass in Bezug auf einen historisierbaren „Verfasser" noch Interpretationsspielraum besteht.

${ }^{3} \mathrm{Zu}$ den bekannten, jüngst von S. Polis, Linguistic Variation in Ancient Egypt: Genres and Registers in the Scribal Repertoire of Amennakhte Son of Ipuy $\left(20^{\text {th }}\right.$ Dynasty), in: J. Cromwell/E. Grossman (Hg.),
Zeit zu Untersuchungen seiner Texte unter verschiedensten Gesichtspunkten ${ }^{4}$. Die Lehre selbst hebt sich innerhalb der Gattung auf verschiedene Weise von den anderen Weisheitstexten ab oder nimmt sich abzeichnende formale Veränderungen auf und führt sie weiter. Elemente klassischer Weisheitstexte, wie sie sich u. a. in der Lehre Ptahhoteps aus dem Mittleren Reich finden - Unterweisung des Schülers, Gehorsam gegenüber Vorgesetzten, Befolgen von Ratschlägen, korrektes Verhalten - werden in der Lehre Amunnachts kombiniert mit Anleihen an Texte der ungefähr gleichzeitig zu datierenden Miscellanies mit Berufscharakteristiken. Die Dialogstruktur, die als neues Stilmittel erstmals am Ende der Lehre Anis bezeugt ist, stellt bei der Lehre Amunnachts das formale Gliederungselement dar. Eine Eigenheit des Textes stellt seine auf das Dorf Deir el-Medine beschränkte Verbreitung dar sowie die zeitlich limitierte Verwendung, mehrheitlich in einem Unterrichtskontext, nicht weit über den Tod Amunnachts hinaus.

Beyond Free Variation: Scribal Repertoires in Egypt from the Old Kingdom to the Early Islamic Period, Oxford (im Druck) zusammengestellten literarischen Texten sind zwei weitere noch unpublizierte hinzugekommen. Siehe G. Burkard, Amunnacht - Scribe and Poet of Deir el Medina: A Study of Ostrakon O Berlin P 14262, in: R. Enmarch/V. M. Lepper (Hg.), Ancient Egyptian Literature. Theory and Practice, Oxford 2013, 61-82 (Ich danke Günter Burkard für die Einsicht in das noch unpublizierte Manuskript) sowie A. Dorn/S. Polis, More Literary Texts of the scribe Amennakhte (in Vorbereitung; O. IFAO inv. OL 117).

${ }^{4}$ Polis, op. cit. (Anm. 3); A. Dorn, Mikrogeschichte und Archäologie: Die Biografie des ägyptischen Schreibers Amunnacht (ca. $* 1190-\dagger 1140$ v. Chr.). 


\section{Kurzer Forschungsüberblick}

In den letzten 20 Jahren hat die bis dahin wenig beachtete Lehre Amunnachts zahlreiche Neubearbeitungen erfahren, sei es durch die Wiederentdeckung eines verschollenen ${ }^{5}$, sei es durch den Fund eines neuen längeren Textträgers, dank dem der Textbestand maßgeblich erweitert werden konnte 6 . In der Folge wurden weitere Parallelversionen gefunden ${ }^{7}$ und mehrere Bearbeitungen trugen $\mathrm{zu}$ einem besseren Textverständnis bei. ${ }^{8}$. Trotz der Erweiterung des Textbestandes sind bisher insgesamt nur fünf Kapitel des wohl um einiges umfangreicheren Weisheitstextes bekannt. Die Unvollständigkeit der Lehre Amunnachts war Anlass für die erneute Beschäftigung mit dem Text und für die Suche nach weiteren Textträgern bzw. nach „missing links“, die zur Zuweisung unbekannter Passagen an die Lehre Amunnachts dienen könnten.

\section{Ein Join von zwei Fragmenten eines literarischen Textes}

Bei der Suche nach der Textfortsetzung ließ sich Ostrakon Kairo CG 25772 (O. CGC 25772) ${ }^{9}$ als die rechte Hälfte von Ostrakon Deir el-Medine 1606 (O. DeM 1606) identifizieren ${ }^{10}$.

S. Bickel/B. Mathieu, L'Écrivain Amennakht et son Enseignement, in: BIFAO 93, 1993, 31-55.

${ }^{6}$ A. Dorn, Die Lehre Amunnachts, in: ZÄS 131, 2004, 38-55.

V. Ritter, Ostraca hiératiques et ostraca figurés. Quelques nouveaux raccords, in: GM 217, 2008, 8384.

${ }^{8}$ P. Dils, oKV 18/3.614+627, Die Lehre des Amunnacht, in: Thesaurus Linguae Aegyptiae (http://aaew.bbaw.de/tla; 27. Juni 2012; Vers 1-78); P. Vernus, Sagesses de l'Égypte pharaonique, Paris 2010², 369-375 (Vers 1-78). P. Grandet, Un document relatif aux grèves de Deîr el-Médînéh en l'an 29 de Ramsès III, et un fragment de l'Enseignement d'Amennakbté, 』 39-48: O. IFAO 1255 A-B, (im Druck). Ich danke Pierre Grandet für die Möglichkeit, sein unpubliziertes Manuskript verwenden zu dürfen.

J. Černý, Ostraca hiératiques (CG 25501-25832), Le Caire 1935, 84 mit Taf. 97* (Umschrift).

${ }^{10}$ G. Posener, Catalogue des ostraca hiératiques littéraires de Deir el Médineh. $\mathrm{N}^{\text {os }}$ 1410-1606. Bd. 3/2 (DFIFAO 20), Le Caire 1978, 80 mit Taf. 52. 52a.
Der Text auf O. DeM 1606 wurde durch H.-W. Fischer-Elfert ${ }^{11}$ vor einigen Jahren inklusive einer partiellen Parallele im Brief auf O. Berlin P 10627, 4-6 $6^{12}$ bearbeitet. Durch den in O. Berlin P 10627,1 erwähnten Adressaten des Briefes, den Schreiber Nachemmut, lässt sich mit der gebotenen Vorsicht zudem eine Datierung in die 1. Hälfte der 20. Dynastie vornehmen, beziehungsweise ein ungefährer Zeitraum bestimmen, während dessen diese literarische Passage in Gebrauch war. Beim Schreiber Nachemmut könnte es sich um den späteren Vorsteher der Mannschaft Nachemmut (vi) handeln, der ab dem 2. Regierungsjahr Ramses' IV. im Amt war; sein Cousin Nachemmut (ii) sowie sein Großvater Nachemmut (i), der zwischen dem 11. und dem 15. Regierungsjahr Ramses' III. Vorsteher der Mannschaft war, lassen sich bei der Identifikation des Briefempfängers jedoch nicht gänzlich ausschließen ${ }^{13}$.

Die beiden neu als zusammengehörig identifizierten Ostraka wurden bei ihrer Edition analog beschrieben: O.CGC 25772 „Tesson de poterie jaune-gris. $-0 \mathrm{~m} .11$ de hauteur", O. DeM 1606 ,Tesson de poterie gris jaunâtre pâle. - 0 m. 101 de hauteur"،. O. DeM 1606, dessen Fragmente 1949 und 1951 im ,grand puits“ (GP), dem großen Brunnenschacht nördlich von Deir el-Medine, gefunden wurden,

${ }^{11}$ H.-W. Fischer-Elfert, Lesefunde im literarischen Steinbruch von Deir el-Medineh (KÄT 12), Wiesbaden 1997, 44-47.

${ }^{12}$ Hieratische Papyrus aus den königlichen Museen zu Berlin. Herausgegeben von der Generalverwaltung. Dritter Band, Leipzig 1911, Taf. 33. 33a. Auf den produktiven Umgang mit der literarischen Passage bzw. auf die Einbettung der literarischen Passage in einen speziellen „literarischen“ Brief und auf dessen mögliche Verwendung ging Fischer-Elfert, op. cit. (Anm. 11), 46-47 bereits ausführlich ein. Von S. Jäger, Altägyptische Berufstypologien (Ling Aeg SM 4), Göttingen 2004, 238-240 wurden die beiden Ostraka im Verhältnis zur Lehre des Cheti besprochen.

${ }^{13} \mathrm{Zu}$ den Personen und den Amtszeiten vgl. die Angaben bei B. G. Davies, Who's Who at Deir elMedina. A Prosopographic Study of the Royal Workmen's Community (EU 13), Leiden 1999, 279 in Verbindung mit chart 7 und den über den Index S. 299 erschließbaren Einträgen. Wahrscheinlich gestützt auf den Namen Nachemmuts (vi) datiert E. F. Wente, Letters from Ancient Egypt, Atlanta 1990, 149 diesen Brief „(Dynasty 20: Ramesses III-IV)“. 
tragen zur Klärung der unsicheren Herkunft „Probablement Biban el-Molouk, fouilles de Th. Davis" von O. CGC 25772 bei. Aufgrund der Herkunft von O. DeM 1606 aus dem ,grand puits“ dürfte auch O.CGC 25772 aus Deir elMedine bzw. der Umgebung von Deir el-Medine und nicht aus dem Tal der Könige stammen.

Das Kairener Fragment komplettiert die Zeilenanfänge von O. DeM 1606. Der Anfang des Textes (oben fehlendes Fragment) fehlt jedoch nach wie vor. Dank O.CGC 25772 lässt sich erkennen, wie auf O. Berlin 10627 der literarische Text in den Brief eingefügt wurde, beziehungsweise wie der literarische Text lautete: $/ / /[p t r]=k b w j r j . t \ldots, / / /$ dich sieht, nicht dringt ein ...". Wie bei anderen Zitaten ebenfalls beobachtbar, macht der neue Textbestand deutlich, dass im Brief an Nachemmut auf O. Berlin P 10627 das Zitat in einem anderen Sinnzusammenhang verwendet wurde ${ }^{14}$. Dort wird der erwähnte Mann als „kein Mann“ bezeichnet, im literarischen Text auf O. DeM 1606 + O. CGC 25772 ist vom „großen Mann“ die Rede.

In Zeile $x+3$ und $x+5$ führt der neue, gemeinsame Textbestand von O. DeM $1606+$ O. CGC $25772 \mathrm{zu}$ inhaltlichen Veränderungen gegenüber der bisherigen Bearbeitung von Fischer-Elfert ${ }^{15}$.
Z. $\mathrm{x}+1:[p t r]=k$ bw jrj.t $m$ d.t $n$ rmt $n b^{c} q m$

Z. $\mathrm{x}+2: m s \underline{d} r=k m-s\}\left[t^{3}\right] y=k$ bj3.t ${ }^{3} . t$ jry $=k n=k r m \underline{t}$

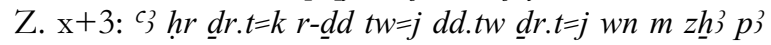
hrj-c

Z. $\mathrm{x}+4: n$ hmmww hr jrj.t ${ }^{c} f d . t s w \underline{d r j}$ t’ g3w.t

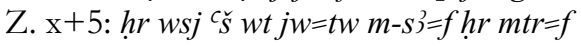

Z. $x+1: / / /$ dich sieht. Niemandes Rede dringt ein in Z. $x+2$ : dein Ohr wegen deines schlechten Charakters. Du hast dich benommen wie ein

Z. $x+3$ : großer Mann wegen deiner Hand: „Ich bin eingesetzt, meine Hand ist eingeweiht in die Schrift" ${ }^{a}$ "، Der Gehilfe

Z. $x+4$ : des Handwerkers (Schreiners) macht eine cfd.t-Kiste. Er verstärkt die $g 3 w . t$-Kiste,

Z. $x+5$ : er sägt Zedernholz ${ }^{b}$ für einen Sarg, indem man hinter ihm her ist und ihn unterweist.

\section{Bemerkungen}

a) Anders als Posener lese ich aufgrund der Konstruktion nicht rdj.tw sondern dd.tw (Stativ). Nach diesem Ausspruch lässt sich - gestützt auf den deutlichen Themenwechsel - ein neues Kapitel ansetzen, das mit „Der Gehilfe ..." beginnt. Als verklammernde Assoziation könnte „meine Hand“ gedient haben, die für das Handwerk des Schreiners Voraussetzung ist, oder aber der Gegensatz großer Mann - kleiner Mann (Gehilfe). Zur Gestaltung von Kapitelübergängen siehe unten.

b) Aufgrund des Determinativs von FischerElfert, op. cit. (Anm. 11), 45 bereits korrekt ergänzt.

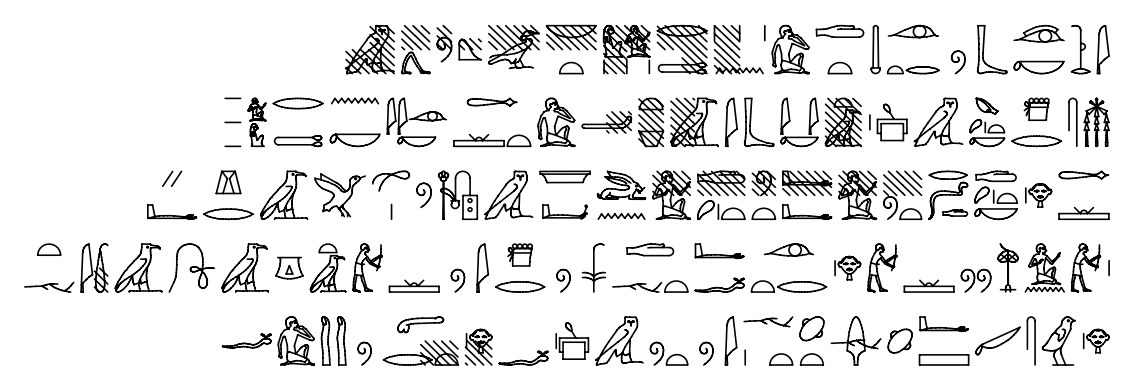

\section{Weitere Textträger der Lehre Amunnachts?}

\footnotetext{
${ }^{14}$ Ein weiteres Beispiel findet sich z. B. auf O. BM EA 50716, siehe dazu unten.

${ }^{15}$ O. CGC 25772 ist nur in Umschrift publiziert (ohne Foto und Zeichnung). Das Ostrakon war zum Zeitpunkt der Bearbeitung des Textes nicht zugänglich, weshalb der Join abgesehen von der Absicherung durch den Paralleltext auf O. Berlin P 10627 hier nicht weiter visualisiert werden kann.
}

Wie eingangs erwähnt, fand sich dieses Passstück bei der Suche nach weiteren Ostraka mit Passagen der Lehre Amunnachts, deren Text im fünften Kapitel abbricht ${ }^{16}$.

${ }^{16}$ Zur Lehre vgl. Dorn, op. cit. (Anm. 6), 38-55. Die einzelnen Kapitel von unterschiedlicher Länge 
Für das Ende der Lehre Amunnachts wurde bereits bei der Neuedition des Textes basierend auf mehreren lexikalischen Bezügen O. DeM 1219 vorgeschlagen ${ }^{17}$, mit ähnlichen Argumenten auch O. DeM 1598 II + O. Michaelides 18 als möglicher Teil der Lehre angesprochen.

Als Arbeitshypothese werden im Folgenden drei weitere Texte mit der Lehre Amunnachts in Verbindung gebracht in der Hoffnung, dass sich in publiziertem oder unpubliziertem Material der eine oder andere „missing link“ eines Tages finden wird, um die Zugehörigkeit abzusichern. Neben den beiden bereits genannten Ostraka sind dies die Texte auf dem hier teilweise vervollständigten O. DeM 1606 + O. CGC 25772, auf O. DeM $1218^{+}$(inkl. Parallelen) ${ }^{18}$ und auf O. DeM $1607^{19}$.

Die Zuweisung der Texte basiert auf materiellen sowie auf thematisch-inhaltlichen Beobachtungen, die einzeln und auch in Kombination Hinweise auf die Zugehörigkeit der

setzten jeweils, abgesehen vom ersten Kapitel, das am Titel ,,angehängt“" ist, auf zumindest einem der Textträger mit einem Rubrum ein, vgl. ebenda, 40-42 (Synopse). Die Kapitel bestehen aus unterschiedlich vielen Versen (1. Kap.: 3 Verse [= Titel] + 18 Verse, 2. Kap.: 14 Verse, 3. Kap.: 10 Verse, 4. Kap.: 18 Verse, 5. Kap.: 15 Verse).

${ }^{17}$ Dorn, op. cit. (Anm. 6), 55. Siehe bereits Fischer-Elfert, op. cit. (Anm. 11), 10-16, speziell seine Bemerkungen S. 12-16 unter Ziffer 6, 8, 15, 16-17, 18 sowie die abschließenden Ausführungen S. 16. Auch Polis, op. cit. (Anm. 3), S 3.5 verbindet O. DeM 1219 mit der Lehre Amunnachts. Von G. Burkard/H. J. Thissen, Einführung in die Altägyptische Literaturgeschichte. Neues Reiches (Einführungen und Quellentexte zur Ägyptologie 6), Berlin 2008, 136-137 wird O. DeM 1219 als eigenständiger didaktischer Text und nicht als möglicher Teil der Lehre Amunnachts aufgeführt.

${ }^{18}$ Besprochen von H.-W. Fischer-Elfert, Eine literarische „Miszelle“. A propos ODeM 1040, 1218 und UC 31905, in: SAK 10, 1983, 151-156. Eine Neubearbeitung mit dem Titel „A literary miscellany. Extended version" ist durch denselben Autor in Zusammenarbeit mit Robert J. Demarée und Vanessa Ritter in Vorbereitung unter Berücksichtigung eines neu identifizierten Fragments zu O. DeM 1218 (vgl. Ritter, op. cit. [Anm. 7], 82) und des Posener bereits bekannten, jedoch unpublizierten Fragments im ägyptischen Museum Kairo (O. Kairo SR 12191), das sich oben an die beiden erwähnten Ostraka aus Deir el-Medine anfügen lässt.

${ }^{19}$ Siehe Fischer-Elfert, op. cit. (Anm. 11), 48-50. jeweiligen Texte zur Lehre liefern können, wobei die Zuweisungen ohne Textüberschneidung hypothetisch bleiben müssen. Durch die Analyse sprachlicher Merkmale eines Schreibers liegt eine weitere Methode vor, die es allenfalls erlaubt, nicht signierte Texte einem Autor zuweisen zu können ${ }^{20}$. Diese lässt sich jedoch erst erfolgreich anwenden, wenn genügend lange Textpassagen vorliegen.

\section{Materielle Argumente}

Tatsache ist, dass der Anfang bzw. die erste Hälfte (s. u.) der Lehre bekannt ist, folglich weitere Textpassagen existieren dürften. Der nachgewiesene Gebrauch in Unterrichtskontexten, der durch das Vorliegen unterschiedlicher Textträger der Lehre Amunnachts auf Ostraka gesichert ist, lässt nicht zugewiesene bzw. nicht identifizierte neuägyptische weisheitlichdidaktische Texte, die ebenfalls in mehr als einer Version vorliegen (O. DeM 1218 ${ }^{+}$; O. DeM 1598 II + O. Michaelides 18), als mögliche Teile der Lehre in Frage kommen. Die Zuweisung solcher Texte an die Lehre gewinnt weiter an Plausibilität, wenn man in Betracht zieht, dass aus der Mitte der 20. Dynastie nur wenige vergleichbare Texte vorliegen ${ }^{21}$. Die Existenz zahl-

${ }^{20}$ Siehe hierzu Polis, op. cit. (Anm. 3).

${ }^{21}$ Die folgende Auflistung nennt einige weitere Fragmente didaktisch-weisheitlicher Texte: O. DeM 1209 (Fischer-Elfert, op. cit. [Anm. 11], 8-9), O. DeM 1217 (H.-W. Fischer-Elfert, Literarische Ostraka der Ramessidenzeit in Übersetzung, Wiesbaden 1986, 5-7), O. DeM 1221, O. DeM 1250 (FischerElfert, op. cit. [Anm. 11], 24-25), O. Fitzwilliam Museum E.68.1926 (F. Hagen, New Kingdom Ostraca from the Fitzwilliam Museum, Cambridge [CHANE 46], Leiden 2011, 8-9 Taf. 5), O. Berlin P 14371 (F. Hintze, Ein Bruchstück einer unbekannten Weisheitslehre, in: ZÄS 79, 1954, 33-36) sowie auch O. Glasgow D. 1925.90 (= HO 40.1, A. G. McDowell, Hieratic Ostraka in the Hunterian Museum Glasgow [The Colin Campbell Ostraka], Oxford 1993, 30-31 mit Taf. 32). Ob unter diesen auch Textträger der Lehre Amunnachts figurieren, kann nicht ausgeschlossen werden. Zudem wurde von Bickel/ Mathieu, op. cit. (Anm. 5), 32 O. Gardiner 341 (= O. Ashm. Mus. 341 bzw. HO 102/4) als möglicher Teil der Lehre vorgeschlagen. Als Argument ließe sich unter anderem das Vorkommen von $d n j$ „Deich“ (Z. 3) 
reicher weiterer noch unbekannter Weisheitstexte, die im Ausbildungskontext Verwendung fanden, erachte ich als gering. Dies lässt sich durch den Befund absichern, dass auf Ostraka nur drei Textanfänge möglicher Weisheitstexte bekannt sind ${ }^{22}$. Anfänge von Texten, die in Ausbildungskontexten rezipiert wurden, können als sichere Indizien für die Existenz eines Textes angesehen werden, da sie am häufigsten als Schreibaufgaben vergeben wurden und folglich die Wahrscheinlichkeit, dass Textträger eines Textbeginns überliefert werden, relativ hoch ist.

Als weiterer Hinweis kann der datierbare produktive Umgang mit Passagen, die hier der Lehre Amunnachts zugewiesen werden und mit der Entstehungs- und Nutzungszeit der Lehre zusammenfallen, angeführt werden, wie er oben für die in O. Berlin P 10627 verwendete Textstelle von O. DeM 1606 + O. CGC 25772 aufgezeigt wurde. Analog verhält sich einer der Texte auf O. BM EA 50716 zu O. DeM 1598 II + O. Michaelides $18^{23}$, auf dem ein Zitat enthalten ist, das den anderen beiden Texten entstammt. Wie bereits auf O. Berlin P 10627 wurde das "Zitat" adaptiert und mit anderem Sinn verwendet. Auch für O. BM EA 50716 lässt sich eine Datierung in die Mitte der 20. Dynastie plausibel machen. Es stammt aus der Arbeiterhüttensiedlung aus der Regierungszeit Ramses' IV. im Tal der Könige, in welcher die Präsenz Amunnachts (v) sowie auch die von Text-

anführen, das in der Lehre Amunnachts zweimal genannt wird: V. 11 und O. DeM $1218^{+}$V. 21. Kritisch im Bezug auf die Zuweisung von O. Gardiner 341 an Amunnacht bzw. an seine Lehre Polis, op. cit. (Anm. 3), § 3.5 .

${ }^{22}$ Zwei nicht zuweisbare Anfänge eines Weisheitstextes „Beginn der Erziehungslehre ///“ liegen in O. Turin 57111 bzw. O. Turin 57134 vso. vor. Sie wurden von Bickel/Mathieu, op. cit. (Anm. 5), 32 versuchsweise mit der Lehre Amunnachts in Verbindung gebracht. Zu erwähnen ist zudem noch das fragmentarische O. Turin 57113 mit der Aufschrift „Beginn der Lehre ///“ auf dem vso. und „,der Schreiber Nachtmin $/ / /$ " auf dem rto.

${ }^{23}$ Zur Bearbeitung der Adaptation siehe H.-W. Fischer-Elfert, Zwei Marginalien zu antikem und ägyptologischem Umgang mit Literatur, in: ZÄS 128, 2001, 86-87, zum Referenztext ders., op. cit. (Anm. 11), 4043. Siehe auch Vernus, op. cit. (Anm. 8), 379 mit Anm. 2. trägern seiner Lehre nachgewiesen ist $^{24}$. Weiter gestützt wird diese Datierung durch paläographische Indizien und durch datierbare Parallelen für die auf dem vso. von O. BM EA 50716 notierten Namenszeichen.

\section{Thematisch-inhaltliche Argumente}

O. DeM 1219, das den möglichen Schluss der Lehre überliefert, weist als charakteristisches Merkmal einen Dialog (Sprecherwechsel LehrerSchüler) auf, der sich auch im gesicherten Textbestand der Lehre Amunnachts findet. Der Dialog als Stilelement in einem Weisheitstext ist im Neuen Reich erstmals am Ende der Lehre Anis bezeugt ${ }^{25}$. Amunnacht nimmt dieses Element auf, wobei er nicht nur das Textende so gestaltet wie Ani, sondern den ganzen Text als Dialog strukturiert.

Am bisherigen Textbestand der Lehre lässt sich zeigen, dass Amunnacht innerhalb des Textes Phrasen, Lexeme und Motive wiederholt aufnimmt und dadurch innertextliche Bezüge schafft, die zur Verklammerung des gesamten Textes führen. Die Gestaltung der Kapitelübergänge hingegen ist sehr locker gehalten. So lassen sich zwischen den ersten vier Kapiteln weder lexikalisch noch thematisch enge Verbindungen feststellen ${ }^{26}$. Dies macht es schwierig, die Position weiterer, nicht direkt anpassender

${ }^{24}$ Zur Herkunft dieses und weiterer Ostraka im British Museum siehe A. Dorn, Maanachtuef, ein (?) einfacher Arbeiter, schreibt Briefe, in: A. Dorn/ T. Hofmann (Hg.), Living and Writing in Deir elMedine. Socio-historical Embodiment of Deir elMedine Texts (AH 19), Basel 2006, 75. Zur Arbeiterhüttensiedlung ders., Arbeiterhütten im Tal der Könige. Ein Beitrag zur altägyptischen Sozialgeschichte aufgrund von neuem Quellenmaterial aus der Mitte der 20. Dynastie (ca. 1150 v. Chr.) (AH 23), Basel 2011.

${ }^{25}$ Auf Bezüge der Lehre zu anderen Texten wird hier nicht weiter eingegangen. Siehe bisweilen Dorn, op. cit. (Anm. 6), 52-55. Eine gesonderte Untersuchung der Bezüge zwischen der Lehre Amunnachts und dem etwa gleichzeitigen P. Lansing ist in Vorbereitung.

${ }^{26}$ Lehrtexte weisen „meist eine eher lockere Struktur auf“ Quack, op. cit. (Anm. 2), 63. Ebenda: „Maßgebend dabei sind lose Assoziationen von Themen und Stichworten, die aufeinanderfolgende Maximen verbinden“. 
Textteile korrekt zu bestimmen. Beim postulierten Textbestand wurden mögliche thematische Bezüge für die Rekonstruktion der Textabfolge berücksichtigt, ohne dass die Position der einzelnen Textteile deswegen als exakt bestimmt gelten kann.

\section{Gesicherter Textbestand der Lehre Amunnachts}

\section{Einleitung $^{27}$}

1 h.j.t- ${ }^{c} m$ sbijy.t mtr.t ${ }^{\bullet}$

2 tz.wn wi.t $n{ }^{c} n \underline{h} \cdot$

3 jrj.n zh' Jmn-nht ${ }^{\circ}$

(4 $\left.[n] \underline{h} r j-{ }^{c}=f H r-M n w\right)$

\section{Kapitel}

$5 \underline{d} d=f t w=k m$ zj $h r s \underline{d} \underline{m} m d . w t{ }^{\circ}$

6 rjwd nfr $r$ bjn ${ }^{\bullet}$

$7 j m h r=k s \underline{d m} m d . w t=j \cdot$

$8 m$-jrj wnj j.d d. $t=j$

$9 n \underline{d m} n \underline{d m} \operatorname{gm}(. w) z j^{\circ}$

$10 \mathrm{jp} m$ k3.t nb.t ${ }^{\bullet}$

$11 \mathrm{jm} \mathrm{hpr} \mathrm{jb=kmj} \mathrm{dnj}{ }^{\circ}{ }^{\circ}$

$12 n t j m w$ wsr.tj $r-g s=f^{*}$

$13 \check{s} z p$ r $3=j m n t j . w=f n b . t{ }^{\circ}$

$14 m-j r h \underline{d n} r z 3 w^{\circ}$

15 j.nw $n$ jr.tj=kj r j3w.t nb.t ${ }^{\circ}$

16 jr.t nb.t $m$ zhi.w ${ }^{\bullet}$

$17^{c} m=k m$ shr.w r-d dd mnh.t.

18 tp-r’.w $d d=j n=k^{*}$

19 m-jr mkh3 md.wt ( ) r’ n jzw.t.

$20 \operatorname{smj}^{C}$ ) nn sw r s.t=f

$21 \mathrm{jm} \mathrm{c}^{3} \mathrm{y} h^{3} \mathrm{itj}=\mathrm{km} \mathrm{s}_{\mathrm{s}}=f^{*}$

$22 m d w=k h r-s\} \quad n j s=\left[t w=f^{*}\right]$

$23 j r y=k z \underline{h}$ ’ $p \underline{h r} r=k p r-{ }^{c} n \underline{h} \cdot$

24 hpr mj hn zhi.w.

2. Kapitel

25 jry $=j m k j j t=j$ hrr n’3y=k smd.t ${ }^{\circ}$

$26 m j h m r[m] j d r=f^{\bullet}$

27 jnk p’ jmw mntk p’ hmw ${ }^{\bullet}$

$28 j . \check{s} m=j$ hr $r$ 3.t $s \underline{d} m n=k^{\bullet}$

29 bw jrj $<=j>$ hrj $n$ shr.w=k${ }^{\bullet}$

$30 t w=j h r m w n d m j=k$ •
1 Beginn der Erziehungslehre,

2 Sprüche für den Weg des Lebens,

3 die der Schreiber Amunnacht gemacht hat

(4 [für] seinen Lehrling Hormin.)

5 Er sagt: Du bist jetzt ein Mann, der Worte hören soll, ${ }^{\mathrm{b}}$

6 um Gutes von Schlechtem zu unterscheiden.

7 Sei aufmerksam, höre meine Worte,

8 und übergehe nicht, was ich dir sage.

9 Es ist sehr angenehm, als Mann befunden zu werden,

10 der fähig ist zu jeder Arbeit.

11 Lass dein Herz zu einem großen Damm werden, 12 neben dem die Flut gewaltig ist.

13 Nimm meine Rede an in all ihren Teilen, 14 weigere dich nicht, sie zu beachten.

15 Blicke mit deinen Augen auf alle Berufe

16 und auf alles, was schriftlich abgefasst wurde.

17 Dann wirst du die Tatsache erkennen, dass die Worte vortrefflich sind,

18 die ich dir gesagt habe.

19 Wende dich nicht ab von den Worten und Sprüchen $^{\mathrm{d}}$ der alten Schriften.

20 Eine heftige Erwiderung ist nicht angebracht.

21 Lass deinen Verstand (Herz) groß sein, wenn er hastig ist.

22 Du sollst nur sprechen, nachdem man aufgerufen worden ist.

23 Du sollst Schreiber werden und im Lebenshaus umhergehen,

24 werde wie eine Bücherkiste.

25 Ich will einen Platz einnehmen, mein Vater, bei deinem smdt-Personal

26 wie ein Esel in ${ }^{\mathrm{e}}$ seiner Herde.

27 Ich bin das Schiff, du bist das Ruder.

28 Ich gehe auf dem (richtigen) Weg, dann höre ich dich.

29 Nicht entferne ich mich von deinen Ratschlägen.

30 Ich bin so auf dem Wasser (im Fahrwasser), wie du es vorspurst.

cit. (Anm. 8), 371-372, die anschließenden Verse sind von H.-W. Fischer-Elfert bereits eingehend besprochen, so dass nur Abweichungen von diesen Bearbeitungen vermerkt wurden. rücksichtung von jüngeren Übersetzungen und Bearbe tungen der Lehre insbesondere von Dils, op. cit. (Anm. 8), Grandet, op. cit. (Anm. 8) und Vernus, op. 


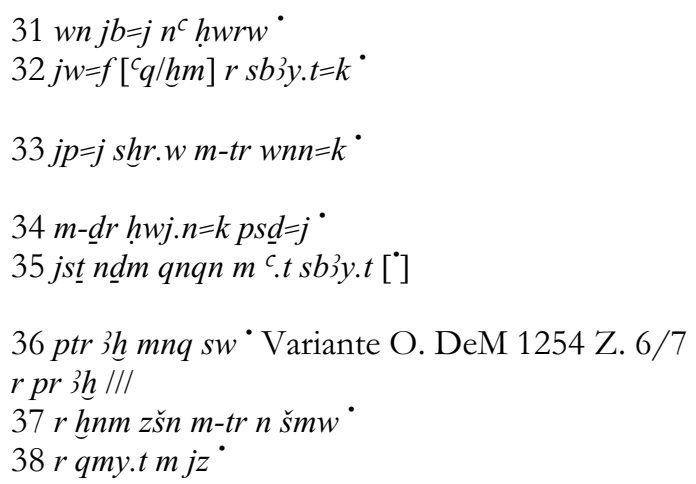

\section{Kapitel}

$393 b . h r=k h r^{c} n h \cdot$

$40 q b(. w)$ nn mtrw

41 jw bw rh $=k$ n' shn.w qn.w.

42 gby $n$ T3-mrj ["]

43 jnj $n=k w^{c}$ hitj $n f r{ }^{\circ}$

$44 r-d n r n 3 y=j s b 3 y . t$

$45 / / / r w j=k m j$ t $p^{3} p^{c}(r) . t^{\cdot}$

46 ntj hr šm.t hrr $w^{c} r<. t>d j d j$

$47 \mathrm{mhr}$ sh.t hft $r$ dj.tw $=f^{*}$

$48 r \underline{d} d j r=j$

\section{Kapitel}

49 mj sdd.w n=kn’ shn.w gỉb.w

50 n hn jw.tj jr.t=f ${ }^{*}$

$51 j w b w s \underline{d} m=f s b 3 y . t n j t^{\circ}$

52 r jrj.t zhij jqr ${ }^{\circ}$

53 sw m pj jmw

$54 j w=f r p^{3} n w h{ }^{\circ}$

$55 \mathrm{hr} t b n=f r p^{3} \mathrm{mw}^{\circ}$

$56 j r y=f w^{c}(r-) h n^{c}\left(^{\circ}\right) m s h . w d b w . w^{\cdot}$

57 zj $n b$ hr ith $n=s n^{\cdot}$

$58 \mathrm{spr} / / / \mathrm{r}$ bnr

$\mathrm{x}+59 / / / n=f \underline{d} . t=f^{\circ}$

$\mathrm{x}+60 t 3 \mathrm{j} / / /$

$\mathrm{x}+61 / / / p 3 k k w$

$\mathrm{x}+62 / / / \mathrm{m}^{3} . \mathrm{w}^{\cdot}$

$\mathrm{x}+63 p 3 w s r$

$\mathrm{x}+64 / / / \operatorname{tr}(?))^{\circ}$

$\mathrm{x}+65 p^{3} z \underline{h} 3(?) h r h r p$

\section{Kapitel}

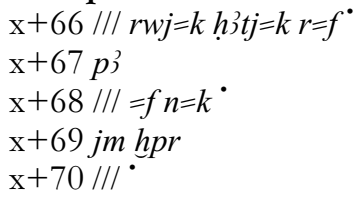

31 Mein Herz war gütig und schwach,

32 jetzt aber ist es deiner Lehre zugewandt ${ }^{\mathrm{g}}$.

33 Ich habe die Ratschläge in der Zeit berücksichtigt, als du da warst,

34 als du meinen Rücken geschlagen hast.

35 Denn es ist angenehm, in der Schule geschlagen zu werden.

36 Siehe, nützlicher ist es, sie (die Schule) zu vollenden ${ }^{\mathrm{h}}$,

37 als das Riechen der Lotosblüten in der Sommerzeit 38 und als Salböl im Grabe.

39 Du sollst dir ein Leben wünschen ${ }^{\mathrm{i}}$, 40 das ruhig ist, ohne beaufsichtigt zu werden, 41 ohne dass du die vielen, beschwerlichen 42 Aufträge Ägyptens kennst.

43 Mach dir ein vollkommenes Herz zu eigen 44 gemäß meinen Unterweisungen.

45 Du sollst [nicht] fliehen wie eine kleine Wachtel, 46 die sich entfernt und hierhin und dorthin flüchtet. 47 Es ist mühsam, im Fangnetz umschlossen zu sein, wenn es geworfen ist, 48 als zu sagen: Ja.

49 Komm, damit man dir die beschwerlichen Tätigkeiten

50 eines uneinsichtigen Dummkopfs erzählt, 51 wenn er die Unterweisungen des Vaters nicht hört, 52 um ein trefflicher Schreiber zu werden.

53 Er (der Schreiber?) ist im Schiff,

54 während er (der Dummkopf?) beim Seil ist, 55 das über seinem Kopf zum Wasser hin ist (i. S. v. verläuft),

56 so dass er eins ist mit den Krokodilen und den Nilpferden.

57 Jedermann zieht zu ihnen hin (?),

$58 / / /$ nach draußen gelangt.

$\mathrm{x}+59 / / /$ für ihn (?) seinen Leib.

$\mathrm{x}+60 \mathrm{Die} / / /$

$\mathrm{x}+61 / / /$ die Dunkelheit

$\mathrm{x}+62 / / /$ Binse (?).

$\mathrm{x}+63$ Der Starke ///

$\mathrm{x}+64 / / /$ Zeit.

$\mathrm{x}+65$ Der Schreiber [?] leitet ///

$\mathrm{x}+66 / / /$ du sollst dein Herz von ihm entfernen.

$\mathrm{x}+67$ Fliegen $/ / /$

$x+68 / / /$ er [?] für dich (?).

$\mathrm{x}+69$ [?] soll werden $/ / /$

$\mathrm{x}+70 / / /$. 
$\mathrm{x}+71$ jm $=k j$ jt.t $j n j j^{\cdot}$

$\mathrm{x}+72$ šbt ///

$\mathrm{x}+73 / / /$ (Zeichenreste) $^{*}$

$\mathrm{x}+74 d n s$ hft $n \underline{d} \underline{\text { d }} \mathbf{d} / / /$

$\mathrm{x}+75 / / /{ }^{\circ}$

$\mathrm{x}+76 m n h t / / /$

$\mathrm{x}+77 / / /^{\circ}$

$\mathrm{x}+78 m r j=s n / / /$ $\mathrm{x}+71$ Du sollst Besitz nicht wegnehmen und bringen.

$\mathrm{x}+72$ Bezahlung (?)///

$\mathrm{x}+73$ (Spuren) $/ / /$

$x+74$ Schwer, wenn [?] fragen ///

$\mathrm{x}+75 / / /$

$\mathrm{x}+76$ Etwas Treffliches ///

$\mathrm{x}+77 / / /$

$x+78$ Sie mögen lieben $/ / /$

Ende des gesicherten Textbestands

Z. 1: ///py.t w[...]n /// s.t ///

Z. 2: wšb wdf $f^{\prime} h d(?) / / /$

Z. 3: /// kj $\underline{d} d . h r=k s b q r=f h n j w . t j / / /$ [ob: $j r . t=f$ oder evtl. $\underline{d} r . t=f ?]$

Z. 4: jw bw rhh=k pj qj $n z \underline{h} \jmath^{\circ}{ }^{c} h^{c} r r w . t y-w r . w$ $c_{s .} . t w=f r$ njs kth. $w^{\cdot}$

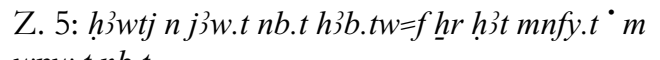
wpw.t nb.t

Z. 6: sbq [sw hr] jr.t hp.w m t3.w nb.w ///

Z. 7: /// ptr wỉh=j shr.w m-bihh[=k]'stp///

\section{Kapitelende?}

\section{Kapitelanfang?}

V. 1: $p t r{ }^{c_{m}=k}{ }^{\circ} m h r b 3 k^{\circ}{ }^{\circ}$

V. 2: $n z j j w . t j \underline{d r} . t=f^{\bullet}$

V. 3: jw wih mtr.w $m-b i h=f^{*}$

V. 4: $m$ tnw $n b^{C_{S} 3}{ }^{\circ}$

V. 5: $r-\underline{d} d d m / / /$

V. 6: $p n^{c} . t w z 3 w h^{c} . t=k \underline{d} s=k$

V. 7: jmj $h r=k r-\underline{h} n w$

V. 8: $m t w=k g b h^{3} t j=k m n f j{ }^{\circ}$

V. 9: $n d m h^{c} . t=k m m n . t \cdot$

V. 10: $h p r=k m j n t j m s=k^{*}$

V. 11: $h n^{c} g s t j{ }^{c} w \cdot t j\left[^{*}\right]^{\times}$

\section{Kapitelanfang}

V. 12: hms $=k m-b 3 h{ }^{\prime} s r . w^{c} 3 . y^{\bullet}$

V. 13: $m \check{s}^{c} t w t . w m-b 3 h=k^{*}$

V. 14: $z[j] n[b] j w=s n{ }^{\circ \text { sic }} h r$ jr.t hn.t $=f^{*}$

V. 15: $m n t=k$ p $3 y=s n$ r $3-\dot{h r j}^{\circ}$

V. 16: jw $=t w h r<\underline{d} d>$ jmj.tw $[r h \underline{c}(?)]$ bjk $n s f^{*}$

V. 17: n3 pds.w r-dr.w wn ${ }^{\bullet}$

V. 18: hrr jr.t ḩsb.w ${ }^{\bullet} n \check{s}^{c}$ tn.t-h.trj ${ }^{\bullet}$

V. 19: $r[d j . t] p 3 y=s n d j . w^{\bullet}$

V. 20: $r f^{3} j=[\operatorname{sn} h r j(?)]=w$ tp ${ }^{\bullet}$

V. 21: wn dnj [jwtn (?)]

V. 22: $m-\underline{h r} r(. t) \operatorname{rnp} . t n\left[^{\circ}\right]$

$\mathrm{V} . \mathrm{x}+22:=w j n q / / /$

V. $x+23: / / /(?)^{\cdot}$

$\mathrm{V} . \mathrm{x}+24:$ smd.t $/ / / n b m-\underline{h} n w=f^{*}$

\section{O. DeM $1218^{+}$(synoptischer Textbestand)}

V. $1^{\mathrm{m}}$ : Siehe, damit du die Mühsal der körperlichen Arbeit

V. 2: eines ungeschickten Mannes verstehst.

V. 3: Wenn Zeugen vor ihn gestellt werden,

V. 4: unzählige an der Zahl,

V. 5: die sagen: $/ / /$.

V. 6: Wende dich um, hüte dich selbst,

V. 7: konzentriere dich auf das Innere(?)

V. 8: und befreie dein Herz von Unrecht.

V. 9: Dein Leib wird sich wohl befinden täglich.

V. 10: Mögest du werden wie der, der dich gebiert,

V. 11: zusammen mit der Palette und der Rolle.

V. 12: Du mögest sitzen ${ }^{\mathrm{n}}$ vor den großen Beamten,

V. 13: und das gesamte Heer ist vor dir.

V. 14: Alle führen seine Aufgaben aus,

V. 15: du aber bist ihr Chef.

V. 16: Wenn man sagt: Gib den, der das Arbeitssoll von gestern kennt.

V. 17: Die Aktenkästen allesamt sind geöffnet

V. 18: beim Ausführen der Abrechnungen des Heeres und der Kavallerie,

V. 19: um ihre Rationen zu verteilen,

V. 20: um zu transportieren/tragen /// Kopf.

V. 21: Dämme/ Register (?) sind ///

V. 22: als Jahresbedarf von ///

V. $\mathrm{x}+22: / / /$ Umarmung (?) ///

V. $x+23: / / /$

V. $\mathrm{x}+24:$ smd.t /// alle /// darin/in seinem Innern. 


\section{Kapitelanfang}

Z. 1: $t w=k^{c}$ 3.tw $h r / / / m$ rdj $h t(?) / / /$

Z. 2: $t w=k$ hnj.tw hr $j b=k h \underline{h r} k m \underline{h r}(?) w p w[. t] / / /$

Z. 3: $n b . t j w=f r j h$ bj3.t bjn.t $r$ ksy ///

Z. 4: hrw $=k j / / / . . / / / . w=k j r y=k \operatorname{srjw} / / /$

Z. 5: $w 3 w . w r=k(?) \underline{d} w n b s h r . w[=k n b . w(?)]$

Z. 6: $n f r$ wr.t /// (?) [tr] $s \underline{d m} / / /$

Z. $7: 3 h^{c}$

Kapitelende?

\section{O. DeM 1607}

Z. 1: Du bist groß in Bezug auf $/ / /$. Gib/Lass nicht //I

Z. 2: Du bist töricht im Bezug auf deinen Verstand (Herz) bei der Durchführung von jedem Auftrag Z. 3: Wozu dient das? Ein schlechter Charakter wird sich beugen $/ / /$

Z. 4: deine Stimme. /// deine ///. Mögest du einen [fähigen (o. ä.)] Beamten abgeben, ///

Z. 5: alles Üble sei fern von dir, [alle deine (?)] Pläne mögen ///

Z. 6: sehr gut sein. /// Zeitpunkt (?) des (?) Hörens $1 / 1$

Z. $7: . .$.

\section{O. DeM 1606 + O. CGC $25772^{28}$}

Z. $\mathrm{x}+1:[p t r]=k b w$ jrj.t $m$ d.t $n \mathrm{rmt} n b^{c} q \mathrm{q}$

Z. $\left.\mathrm{x}+2: m s \underline{d} r=k m-s^{3}[t]\right\} y=k b j 3 . t^{c}$ 3.t jry $=k n=k r m \underline{t}$

Z. $\mathrm{x}+3:{ }^{c}, \underline{h} r \underline{d r} \cdot t=k r-\underline{d} d t w=j d d . t w \underline{d} d r . t=j$ wn $\left.m z \underline{h}\right\}$

Z. $x+1: / / /$ dich sieht. Niemandes Rede dringt ein in Z. $x+2$ : dein Ohr wegen deines schlechten Charakters. Du hast dich benommen wie ein

Z. $x+3$ : großer Mann wegen deiner Hand: „Ich bin eingesetzt, meine Hand ist eingeweiht in die Schrift"

\section{Kapitelende}

\section{Kapitelanfang}

Z. $\mathrm{x}+3$ (Fortsetzung:) p3 $\mathrm{hrj}^{\mathrm{c}} \mathrm{c}$

Z. $\mathrm{x}+4: n$ hmmww hr jrj.t ${ }^{c} f d$.t $s w \underline{d}$ rj $t 3$ g3w.t

Z. $\mathrm{x}+5: h r w s j c^{c} w t j w=t w m-s \jmath=f h r m t r=f$

\section{Z. $x+3$ : Der Gehilfe}

Z. $\mathrm{x}+4$ : des Handwerkers (Schreiners) macht eine ${ }^{c} f d . t$ Kiste. Er fertigt mühselig die $g 3 w . t$-Kiste an und Z. $x+5$ : er sägt Zedernholz für einen Sarg, indem man hinter ihm her sein muss und man ihn unterweist.

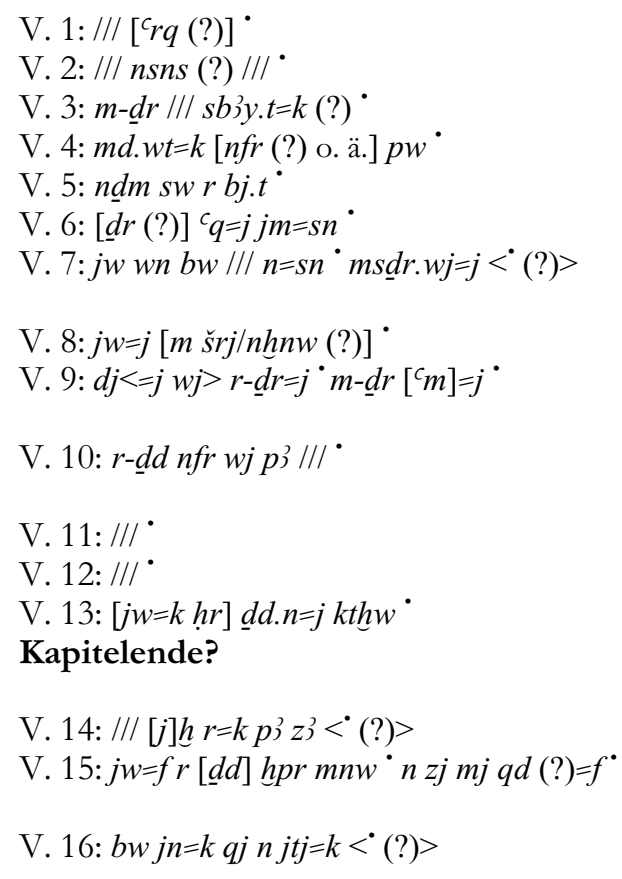

\section{O. DeM 1219}

V. 1:///

V. 2: ///

V. 3: als/seit [... ich hörte (?)] deine Lehre.

V. 4: Deine Worte sind [perfekt (o. ä.)].

V. 5: Süßer sind sie als Honig,

V. 6: [seit (o. ä.)] ich in sie eingedrungen bin,

V. 7: als es einen Ort gab /// für sie <in? > meinen

Ohren,

V. 8: als ich [noch ein Kind] war.

V. 9: <Ich> gab mich ihnen gänzlich hin, als ich [erkannte]:

V. 10: „Wie gut ist der dran, [der auf sie hört (o. ä.)"] //I

V. 11: ///

V. 12:///

V. 13: [und du] mir andere (Sprüche) zitierst ///

V. 14: (Vater:) [Was] soll das, du Sohn (?)

V. 15: Er [sagt (?)]: „Das Denkmal eines Mannes (von Rang) ist [sein] Charakter".

V. 16: Du holst das Wesen deines Vaters nicht ein

${ }^{28}$ Kommentare zum Text finden sich eingangs des Artikels. 


\author{
V. 17: [m šs]m.tjwj.t ${ }^{\bullet} m j w . t j \underline{d r . t}=k^{\bullet}$ \\ V. 18: $b[w r h]=k<r(?)>k 3 . t n b<. t>$ \\ V. 19: jw=j hpr [m(?)] bn jb=j $m$ h.t $t=j{ }^{\bullet}$ \\ V. 20: $s \underline{h} 3=j \underline{t z} . w j r . n=k$
}

\section{Bemerkungen}

a) Vers vier gehört nicht zum Kernbestand des Textes. Er lässt sich als optionaler Vers bezeichnen, der eine Widmung/Zueignung des Textes enthalten kann. Die einzige Widmung ,[für] seinen Lehrling Hormin" findet sich auf O. BM EA 41541. Bei der Interpretation der Lehre sollte die Widmung nicht stillschweigend für alle übrigen Textträger mitgedacht werden, wie dies bei den Übersetzungen, die auf dem synoptischen Textbestand beruhen, meist der Fall ist. Im Bezug auf die Kontextualisierung der einzelnen Textträger ist diese Beobachtung von Bedeutung. Bei O. BM EA 41541, das von Amunnacht selbst geschrieben worden sein könnte - oder setzt sich Hormin selbst in die Lehrlingsrolle? richtet sich der Text an Hormin, weitere Textträger entstehen unter Anleitung Amunnachts (?) im Ausbildungskontext unter bewusster Weglassung einer Zueignung.

b) Auf Deutsch lässt sich die vorliegende Konstruktion nicht befriedigend wiedergeben, vgl. die wörtliche Übersetzung, ,du bist von der Art (bzw. als) Mann (bzw. du bist in der Situation von jemandem), beim Worte hören (Worte zu hören)“.

c) Vgl. die Untersuchungen zu gmj unter Berücksichtigung der vorliegenden Passage von P. Vernus, Le verbe $g m(j)$ : essai de sémantique lexicale, in: E. Grossman/S. Polis/J. Winand (eds.), Lexical Semantics in Ancient Egyptian (LingAeg SM 9), Hamburg 2012, 387-438, spez. 419-420.

d) Als Singular notiert.

e) Je nach Übersetzung von $h r$ in V. 25 entsteht ein stark abweichender Sinn. Die hier vorgenommene mit „bei“ führt zur Unterordnung des Schülers wie im übernächsten Vers 27 (ich bin das Schiff, du bist das Ruder). Bei einer Übersetzung von $h r$ als „über“ rückt der Schüler dem Vater in seiner Funktion nach. Auf O. Lacau (HO III/3) besteht im Text von Vers 26 eine kleine Lücke vor $j d r=f$,,seine Herde“, in der sich $h r$ oder $m$ ergänzen lässt. Mit $m$ ,in“ würde die Unterordnung weitergeführt, mit $h r$ das gegenteilige Bild.

f) Mit Vernus, op. cit. (Anm. 8), 372. 374, der $d m$ mit der Bedeutung von „,stechen (,percer“, ici ,tracer un sillage dans l'eau“")" ansetzt. Mit der Verwendung von $d m$,nennen“ lautet der Vers „Ich bin auf dem Wasser des dich Nennens" i. S. v. ich mache deinen
V. 17: [durch Hin-] und hergehen, durch

Ungeschicklichkeit.

V. 18: Du kennst keine Arbeiten ${ }^{\circ}$.

V. 19: (Sohn): Ich bin geworden [zu einem (?)]

„Mein Herz ist nicht in meinem Leibe.“

V. 20: Ich denke über die Sprüche nach, die du verfasst hast.

Namen berühmt, siehe Dils, op. cit. (Anm. 8). Inhaltlich entsteht so ein enger Bezug zu Vers 28 „Ich gehe auf dem Weg des auf dich Hörens“. Bei der Interpretation von $d m$,nennen“ bleibt jedoch unberücksichtigt, dass auf keinem Textträger das Determinativ des Mannes mit der Hand am Mund notiert wurde.

g) Zu den Zeichenresten an dieser Stelle siehe Dorn, op. cit. (Anm. 6), Taf. IV Linie 10. Beide vorgeschlagenen, inhaltlich passenden Ergänzungen - H.-W. Fischer-Elfert, „Ich bin das Schiff - du bist das Ruder", in: SAK 11, 1984, 336-337 und Dils, op. cit. (Anm. 8), \32 mit Anmerkung - lassen sich nicht zweifelsfrei mit den Zeichenresten verbinden. Die gegebene Übersetzung kommt ohne das fehlende Wort aus.

h) Die Variante auf O. DeM $1254 r$ pr ih ,zum/als nützliches Haus“ macht inhaltlich wenig Sinn.

i) Die Bearbeitung eines neu von Vanessa Ritter identifizierten Textzeugen (vgl. Ritter, op. cit. [Anm. 7], 83-84) durch Grandet, op. cit. (Anm. 8) führte zu einem besseren Verständnis einiger Passagen der Verse 39-48.

j) In Analogie zu V. 50 ist statt ,der Tor, der Ungeschickte“ auch „un[einsichtiger] Dummkopf“" möglich.

k) Ich verbinde $w r . w$,die Großen“ mit dem vorangehenden rw.ty „Doppeltor“: „Doppeltor der Großen“. Anders Fischer-Elfert, op. cit. (Anm. 11), 41-42 sowie auch Jäger, op. cit. (Anm. 12), 300 b mit Anm. 412, die wr.w als Adjektiv auffassen. Das Determinativ (A 1) spricht jedoch dagegen. Für rw.ty „Doppeltor" als Ort, wo die Großen hingehen, finden sich ab dem Mittleren Reich verschiedene Belege, siehe die Angaben im digitalen Zettelarchiv (Thesaurus Linguae Aegyptiae, http://aaew.bbaw.de/tla).

1) $\mathrm{Zu}$ dieser Stelle inklusive der Ergänzung $s w$ hr in der Lücke auf O. DeM 1598 Zeile 6 siehe Jäger, op. cit. (Anm. 12), 300-301. Die Wendung wpw.t sbq[.t] findet sich auch als wpw.t c3.t sbq[.t] in P. Gurob, siehe A. H. Gardiner, RAD 14,8-9.

m) Ich danke Robert J. Demarée für die Erlaubnis, die ersten fünf Verse dieses Kapitels hier wiedergeben zu dürfen. Die Zuweisung von O. Kairo SR 
12191 an O. DeM 1218 geht auf G. Posener zurück. Die Transkription des Ostrakons, u. a. mit diesen fünf Versen, wurde jedoch erst 2006 durch Rob Demarée erstellt. Auf ein in die Bruchstelle zwischen diesen beiden Ostraka gehörendes Fragment hat Vanessa Ritter hingewiesen (hier nicht enthalten) ${ }^{29}$. Für die Vorlage der hier wiedergegebenen Textstelle konnte ich auf ein Rohmanuskript zurückgreifen (siehe hierzu die Angaben oben in Anm. 18), das mir freundlicherweise Hans-Werner Fischer-Elfert zur Verfügung gestellt hat.

n) Im Gegensatz zu Fis cher-Elfert, op. cit. (Anm. 18), 152 setze ich hier nicht nur einen neuen Satz an, sondern lasse mit dem prospektiven $s \underline{d} m=f \quad h m s j=k$ auch ein neues Kapitel beginnen. Gestützt wird dies formal u. a. durch das nochmalige Schreiben des „Kapitelanfangs“ auf dem vso. ca. ein halbes Jahr später als die übrigen Textpassagen auf dem Ostrakon (zu den Daten siehe die Angaben unten).

Die Kapitelverklammerung wurde hier durch die Thematisierung des Schreiberberufes realisiert: Das Kapitelende beschreibt das moralisch korrekte Verhalten eines Schreibers, das folgende Kapitel setzt ein mit Schilderungen zum „Schreiber bei der Arbeit".

o) Statt $b[h]$ ergänze ich parallel zu Vers $16 b w+$ Verb: $b[w r h]=k$.

\section{Verklammerungen im Text}

Wiederholt finden sich verschiedene Lexeme für das Wort „Kiste“, das sich wie ein roter Faden durch den Text zieht: Z. 24 (hn zh hi.w = Bücherkiste); O. DeM $1218^{+}$V. 17 (pds-Kasten, Aktenkästen); O. DeM 1606 x+4 ('fd.t-, gjw.tKiste).

Als Opposition zu den Kisten, die zweimal klar mit Schriften in Verbindung stehen (Ort der Wissensspeicherung), lässt sich das Wort für „Törichter/töricht" $h n$ anführen, das ebenfalls mehrfach im Text verwendet wurde. In der ersten Passage kommt $h n$ in der Verbindung mit $j w . t j \quad j r . t=f$,uneinsichtig“ (V. 50) vor, auf O. DeM 1598 II + O. Michaelides 18 Z. 3 findet sich nach $h n$ eine Zerstörung. Mit $j w . t j \underline{d} r . t=f$ bzw. $=k$,ungeschickt" findet sich mit derselben Konstruktion in O.DeM $1218^{+}$V. 2 und in O. DeM 1219 V. 17 ein weiterer Ausdruck aus diesem Sinnbereich. Gemeinsam mit hn bildet

${ }^{29}$ Ritter, op. cit. (Anm. 7), 82 (O. DeM inv. 2828). „das Dumme (uneinsichtig, ungeschickt)“ eine Opposition zum Ansehen (Wissen) des Schreiberberufs.

Einer der zentralen Begriffe der Lehre stellt „Herz" (jb bzw. hijtj) dar, der sich im Kernbestand des Textes fünfmal findet, in der Fortsetzung weitere dreimal ${ }^{30}$. Es fällt auf, dass die beiden Termini bei den ersten vier Nennungen alternierend verwendet werden. Neben dem Herz sind die Ohren (ms $d r)$ bzw. das mit ihnen ausgeführte Hören $(s \underline{d} \underline{\mathrm{d}})$ als Sinnesorgan ein weiteres Charakteristikum des Textes, wobei dies angesichts der vorliegenden Gattung - Unterweisungen, die der Schüler zu hören und anzunehmen hat - nicht erstaunt, weshalb diese Beobachtung, wie die ebenfalls zahlreiche Verwendung andere Termini ( $\underline{d} d$,sprechen“, $m d . t$ „Rede“, $r$ ' „Mund“; $z j$ „Mann“) als gattungsimmanent zu klassifizieren sind und deshalb nicht geeignet sind, um aufgrund ihres Vorkommens in einem nicht zugewiesenen Textfragment eine Zuweisung vorzunehmen.

\section{Wiederaufnahme einzelner Lexeme und Inhalte - Kapitelübergänge}

Zweimal findet sich im Text bj3.t, einmal als bj3.t bjn (O. DeM 1607 Z. 3) einmal als bj3.t C3.t (O. DeM 1606 + O. CGC 25772) und zwar zur Bezeichnung eines schlechten Charakters ${ }^{31}$. Bei der vorgeschlagenen Textabfolge inklusive der Kapitelgliederung würden mit bj3.t in Verbindung mit $s \underline{d} m$, hören“ bzw. ms $\underline{d} r$ „Ohr“ zwei Bezüge zu aufeinander folgende Kapitel vorliegen. Der Ausdruck bj3.t 93.t ist zudem in

${ }^{30}$ Zur Bedeutung der beiden Termini in der Literatur des 2. Jahrtausends v. Chr. unter Berücksichtung der Lehre Amunnachts vgl. M. I. Toro Rueda, Das Herz in der ägyptischen Literatur des zweiten Jahrtausends v. Chr. Untersuchungen zur Idiomatik und Metaphorik von Ausdrücken mit $j b$ und $h 3 t j$ unter htp://webdoc.sub.gwdg.de/diss/2004/toro_rueda/; siehe zudem R. Nyord, Breathing Flesh. Conceptions of the Body in the Ancient Egyptian Coffin Texts (CNI Publications 37), Kopenhagen 2007.

${ }^{31}$ Siehe hierzu die Angaben von Fischer-Elfert, op. cit. (Anm. 11), 49 mit weiteren Verweisen sowie Bickel/Mathieu, op. cit. (Anm. 5), 40-41 mit Anm. 66. 
zwei weiteren Texten Amunnachts belegt: in O. Gardiner 25 vso. Z. 6 (= O. Ashm. Museum 25) und in O. Turin 57002 vso. 5. Auf die Verwendung von ${ }^{c}$, groß ${ }^{\prime}$ als mögliches Stilmittel in Texten Amunnachts haben Bickel/Mathieu hingewiesen ${ }^{32}$.

Das Wortpaar $c^{c} q$,eindringen“ und $m s d r$ „Ohr“ könnte als Verklammerung zweier Kapitel (O. DeM 1606 + O. CGC $25772 \mathrm{x}+1$ ) gedient haben, wobei sich ein partieller Parallelismus beobachten lässt, bei dem beim Wiederaufgreifen $m s \underline{d} r$, Ohr" den Vers sprengend erst im folgenden Vers vorkommt (O. DeM 1219 V. 6-7).

Das eingangs (V. 11) verwendete $d n j^{33}$ ist unten in O. DeM $1218^{+}$V. 21 erneut belegt, ebenso $s m d . t$ (V. 25) in O. DeM $1218^{+}$V. $\mathrm{x}+24$. Beiden Begriffen gemeinsam ist ihre Verwendung auch in anderen literarischen Texten (mehrfach in den Miscellanies), was ihre Nutzung als Argument für eine Textzuweisung abschwächt.

Auffallend dicht sind die Bezüge in O. DeM 1219 zum Kernbestand der Lehre. Bereits Posener $^{34}$ hat auf die inhaltliche Verwandtschaft von V. 17-18 zu V. 9-10 auf O. DeM 1219 hingewiesen, etwas unsicherer ist aufgrund des nicht ganz erhaltenen Textbestands der Bezug zwischen V. 32-33 und den V. 6-8 von O. DeM 1219. Auffällig ist hingegen die zweimalige, je anders ausgedrückte Nennung von „Unstetigkeit": V. $\mathrm{x}+71$ (des Kernbestands) und in V. 17 auf O. DeM 1219. In V. 18 von O. DeM 1219 könnte ein Bezug zu V. 10 der Lehre vorliegen, wo ebenfalls ,jede Arbeit" genannt ist. Der Schlusssatz bzw. sogar die letzten beiden Verse von O. DeM 1219 lassen sich leicht mit dem Anfang der Lehre V. 2-3 verbinden bzw. als Antwort auf die Verse 17-18 verstehen, wobei der Schluss „Ich denke über die Sprüche nach, die du verfasst hast", so allgemein sind, dass sie jede „erfolgreiche“ Unterweisung abschließen können.

\footnotetext{
${ }_{32}$ Bickel/Mathieu, op. cit. (Anm. 5), 48.

${ }_{33}$ Siehe auch die Angaben oben am Ende von Anm. 21.

${ }^{34}$ G. Posener, L'exordre de l'instruction éducative d'Amennakhte (recherches littéraires, V), RdE 10, 1955, 67.
}

Sowohl in O. DeM 1598 II + O. Michaelides 18 als auch in O. DeM $1218^{+}$wird auf das Militär Bezug genommen, in der ersten Textpassage kurz, in der zweiten etwas länger, wobei der Schreiberberuf, ohne dass eine eigentliche Soldatencharakteristik vorliegt, über den Militärstand gestellt wird.

\section{Länge der Lehre}

Falls die Zuweisung von O. DeM 1607 an die Lehre Amunnachts zutrifft und der Vermerk $n t j$ $r$ mh 11 ,Was macht das 11. (Kapitel) ${ }^{635}$ auf dem Rand sich ebenfalls auf diesen Text bezieht, so liefert er einen Anhaltspunkt zur Gesamtlänge der Lehre: Der Textbestand von bis jetzt fünf Kapiteln mit durchschnittlich 15 Versen wäre sicher zu verdoppeln. Betrachtet man die Länge der mit der Lehre in Verbindung gebrachten Texte, so dürften zu den fünf Kapiteln der Lehre weitere sechs, wenn nicht sogar acht Kapitel hinzukommen ${ }^{36}$ :

O. DeM 1598 II + O. Michaelides 18: 1 Kapitel à ca. 14 Versen, Anfang zerstört, Textende fällt wahrscheinlich mit einem Kapitelende zusammen;

O. DeM 1218 ${ }^{+}: 1$ evtl. 2 Kapitel, wobei das 2. mit $h m s j=k$ einsetzt und zwar an der Stelle, die auf O. DeM 1218 rto. mit einem Kreuz markiert wurde und auf dem vso. von O. Kairo SR 12191 (an O. DeM 1218 anpassend) von einer anderen Hand nochmals notiert wurde;

O. DeM 1607: 1 Kapitel à ca. 11 Versen, das mit einem Kapitelanfang einsetzt;

O. DeM 1606 + O. CGC 25772: 2 Kapitel. Ende eines Kapitels in Z. $x+3$, Beginn eines zweiten Kapitels;

O. DeM 1219: entweder langer Schluss der Lehre mit Sprecherwechsel, bestehend aus 20 Ver-

\footnotetext{
${ }^{35}$ Fischer-Elfert, op. cit. (Anm. 11), 50.

${ }^{36}$ Da keiner der Textträger Rubra aufweist, ist eine exakte Bestimmung der Kapitelanfänge bzw. -längen nicht eindeutig vorzunehmen. Bei intaktem Textbeginn ist die Wahrscheinlichkeit groß, dass der Schülertext mit dem Beginn eines Kapitels einsetzt. Die Anzahl Verse sowie die Wechsel von Themen stellen weitere Hilfsmittel zur Bestimmung von Kapiteln dar.
} 
sen, oder Beginn des letzten kurzen Schlusskapitels mit Vers 14.

\section{Abfassungsdatum der Lehre}

Auf dem vso. von O. Lacau (= HO III/3) das rto. enthält das zweite, dritte und Teile des vierten Kapitels der Lehre - befindet sich die Datumsangabe rnp.t-zp 26 3bd 1 pr.t sw 13 /II „26. Regierungsjahr 1. Monat der Peretjahreszeit Tag $13 / / / “$. In Verbindung mit den bekannten Lebensdaten des Autors Amunnacht - geboren einige Jahre vor der Thronbesteigung Ramses' III., gestorben im 6. oder 7. Regierungsjahr Ramses' VI. ${ }^{37}$ - handelt es sich bei diesem Jahr 26 um das 26. Regierungsjahr Ramses' III. Weitere Daten finden sich auf O. DeM 1219, das den möglichen Schluss der Lehre enthält $m h \mathrm{~m}$ rnp.t-zp 25 ibd 4 šmw sw 18 ,,vollendet ${ }^{38}$ im 25. Regierungsjahr 4. Monat der Schemujahreszeit Tag 18“, sowie auf dem vso. von O. Kairo SR 12191, eines der an O. DeM 1218 anpassenden Fragmente, das ebenfalls mit der Lehre in Verbindung gebracht wurde. Auf diesem sind sogar zwei Daten enthalten. Das erste liegt gerade mal fünf Tage vor dem Datum unter dem möglichen Schluss der Lehre auf O. DeM 1219 rnp.t-zp 25 3bd 4 šmw sw 13 ,25. Regierungsjahr 4. Monat der Schemujahreszeit Tag 13“, das zweite Datum stammt aus dem folgenden Regierungsjahr rnp.t-zp 26 3bd 2 pr.t sw 10 ,26. Regierungsjahr 2. Monat der Peretjahreszeit Tag $10^{66^{39}}$. An den hier gemeinsam betrachteten Texten fällt auf, wie häufig sich eine Jahresangabe findet. Von McDowell wurden 110 literarische Ostraka mit Datumsvermerken zusammengetragen $^{40}$. Von diesen weisen nur gerade acht nicht

${ }^{37}$ Davies, op. cit. (Anm. 13), 115 mit Anm. 421.

${ }^{38} \mathrm{Zu}$ diesem Vermerk vgl. die Ausführungen von Fischer-Elfert, op. cit. (Anm. 11), 15-16 ,eine Abschrift und/oder einen Text vollenden/beenden“, der diese Angabe vorsichtig als Schluss des Textes und nicht eines Kapitels taxiert.

${ }^{39}$ Weitere Datumsvermerke, jedoch ohne Angabe eines Regierungsjahres, finden sich auf Textträgern der ersten beiden Kapitel der Lehre, vgl. Dorn, op. cit. (Anm. 6), 40-41.

${ }^{40}$ A. McDowell, Student Exercises from Deir elMedina. The Dates, in: P. Der Manuelian (Hg.), nur Monats-, sondern auch Jahresangaben auf, wobei O. DeM 1219 und O. Lacau (= HO III/3) darin bereits enthalten sind. Unter Berücksichtung von O. Kairo SR 12191 liegen aus Deir el-Medine neun Ostraka mit Jahresangaben vor, von denen drei auf möglichen Textträgern der Lehre Amunnachts notiert wurden.

Bereits durch O. Lacau (= HO III/3) lässt sich das Abfassungsdatum der Lehre Amunnachts für das 26. Regierungsjahr Ramses' III. belegen, durch die neu mit der Lehre in Verbindung gebrachten Passagen dürfte der Text der Lehre bereits im 25. Regierungsjahr Ramses' III. vollständig vorgelegen haben.

Durch die Notizen zu den Streiks auf dem rto. und dem vso. von O. IFAO 1255 - genannt sind die Tage 10-18 des 2. Monats der Peretjahreszeit - lässt sich das Abfassungsdatum dieses Ostrakons durch den Abgleich mit Vermerken zu denselben Tagen auf dem Streikpapyrus (P. Turin 1880), der im 29. Regierungsjahr Ramses' III. verfasst wurde, mit diesem Jahr verbinden.

\section{Intertextualität auf Textträgern}

Auf fünf der über 20 bekannten Textträger, inklusive denen des neuen Textbestands, wurden auch andere Texte notiert. Auf O. München ÄS $396^{41}$ findet sich auf dem rto. der Beginn der Lehre des Cheti (Kapitel 1-2) und auf dem vso. der Beginn der Lehre Amunnachts. Die Lehre Chetis lässt sich geradezu als Pate für die jüngere Lehre Amunnachts bezeichnen, in welcher durch die Erwähnung ,aller Berufe“ in V. 15 und die enthaltenen Berufscharakteristiken Bezüge zur Lehre des Cheti geschaffen werden. Auf dem mit wenig Text beschrifteten Ostrakon JdE 96397 stehen die ersten 4 Verse der Lehre Amunnachts sowie Teile des Beginns der Lehre

Studies in Honor of William Kelly Simpson, Boston 1996, 601-608, spez. 603-604.

${ }^{41}$ Siehe J. v. Beckerath, Ostrakon München ÄS 396, in: SAK 10, 1983, 63-69; Jäger, op. cit. (Anm. 12). 
des Amenemhat ${ }^{42}$. Auf dem vso. von O. Lacau (HO III/3) sind Reste eines bisher nicht identifizierten literarischen Textes (punktiert) enthalten.

O. IFAO 1255 trägt als Hauptaufschrift auf rto. und vso. Aufzeichnungen zu den Ereignissen während der Streiks der Arbeiter von Deir el-Medine im Jahr 29 Ramses' III. Um $90^{\circ} \mathrm{zu}$ diesen Notizen gedreht, finden sich auf dem vso. die Verse 39-48 der Lehre Amunnachts, die aufgrund der Disposition vor der Aktennotiz des vso. niedergeschrieben worden sein dürften. Inhaltlich ist der Bezug der notierten Passage der Lehre Amunnachts zu den Ereignissen der Streiks beachtenswert: V. 39f. „Du sollst dir ein Leben wünschen, das ruhig ist, ohne beaufsichtigt zu werden", in V. $43 \mathrm{ff}$. folgt dann die im Verständnis nicht ganz einfache Passage mit der fliehenden Wachtel im Fangnetz. Die neben dem administrativen Text notierten Verse gewinnen durch die Vermutung von P. Grandet, dass das Ostrakon von Amunnacht selbst geschrieben wurde, weiter an Relevanz: Ihre Niederschrift lässt sich als literarischer Kommentar zu den Streiks durch den Schreiber Amunnacht, der die Streiks „privat" dokumentierte, taxieren $^{43}$.

Auf dem neu mit der Lehre Amunnachts in Verbindung gebrachten O. DeM 1598 befindet sich oben der ganz in rot geschriebene fragmentarische Text (I) „Die beiden Schakale ${ }^{\text {‘4 }}$, darunter in schwarz vielleicht von derselben Hand die Verse des Weisheitstextes mit einem „Lobpreis auf den Schreiberberuf“" (II). Einen Bezug zwischen den beiden Texten - kopulierende Schakale (I) und Schreibercharakteristik (II) - vermag ich nicht zu erkennen, was jedoch durch die Kürze des fragmentarisch erhaltenen Textes (I) bedingt sein könnte.

\section{Summary}

The newly found join of O. DeM 1606 with O. CGC 25772 allows to add a new fragment to the "reproach with the following description of a carpenter's job" as well as to see how a part of this text was inserted in the "literary" letter on O. Berlin P 10627. It is then proposed as a working hypothesis that O. DeM 1606 + O. CGC 25772 were part of the teaching of Amennakhte as well as O. DeM 1598 II with a parallel on O. Michaelides 18, O. DeM $1218^{+}$ with parallels, O. DeM 1607, and O. DeM 1219.

\section{Keywords}

instruction of Amennakht - literature NK quotation - textual reconstruction
${ }^{42}$ Bei Dorn, op. cit. (Anm. 6), 39-40 noch als O. Kairo ohne Nummer aufgeführt.

${ }^{43} \mathrm{Zu}$ dieser Klassifikation des Streikpapyrus siehe M. Müller, Der Turiner Streikpapyrus (pTurin 1880), in: B. Janowski/G. Wilhelm (Hg.), TUAT NF 1, Texte zum Rechts- und Wirtschaftsleben, Gütersloh 2004, 165-184.

\footnotetext{
${ }^{44}$ Fischer-Elfert, op. cit. (Anm. 11), 160-163.
} 
HENNING FranZMEIER und ANKE WEBER

\title{
Die ZÄS nach dem Zweiten Weltkrieg: Der Antrag auf Lizenzerteilung vom 27.01.1947
}

\author{
Hierzu Tafel XVII-XVIII
}

Bis 1944 gehörte die Zeitschrift für Ägyptische Sprache und Altertumskunde (ZÄS) dem traditionsreichen Leipziger Verlag J. C. Hinrichs $^{2}$. Die erste Ausgabe der ZÄS nach dem Krieg erschien erst im Jahre 1954 nach einer Unterbrechung von zehn Jahren im (Ost-) Berliner Akademie-Verlag in Arbeitsgemeinschaft mit dem später liquidierten Leipziger Verlag J. C. Hinrichs ${ }^{3}$. An dieser Stelle soll ein vorangegangener, zunächst erfolgloser Versuch aus dem Jahr 1947 dokumentiert werden, die ZÄS wieder neu aufzunehmen. Im Zentrum steht dabei der Antrag auf Erteilung der Lizenz für die Neuaufnahme der ZÄS aus der Feder des mit dem Verlag eng verbundenen Hermann Grapow ${ }^{4}$, der sich an die sowjetische Militäradministration richtete ${ }^{5}$. Im Rahmen dieses kurzen Artikels soll die Argumentation vorgeführt werden, die insbesondere vor dem Hintergrund der gegebenen Rahmenbedingungen sehr aufschlussreich für das Selbstverständnis der deut-

${ }^{1}$ Sächsisches Staatsarchiv, Staatsarchiv Leipzig, 22208 J. C. Hinrichs Verlag, Leipzig, Nr. 636, Bl. 33-34.

${ }^{2} \mathrm{Zu}$ dem Verlag siehe Geist 1991 sowie Franzmeier, Weber 2013, 116-118.

${ }^{3}$ Der Verlag J. C. Hinrichs erhielt in der Tat nie wieder eine Drucklizenz, sondern lediglich eine Verkaufslizenz. So wurden etwa die Rechte an älteren Publikationen, wie etwa den „Urkunden“ und dem „Wörterbuch der ägyptischen Sprache“, verkauft.

${ }^{4}$ Sächsisches Staatsarchiv, Staatsarchiv Leipzig, 22208 J. C. Hinrichs Verlag, Leipzig, Nr. 636, B1. 3334. Siehe zu der Verbindung zwischen dem Verlag und Hermann Grapow Franzmeier, Weber 2013, $133-$ 141.

Auf die Struktur der sowjetischen Militäradministration und die Bedingungen der Lizenzierung von Druckerzeugnissen und Verlagen soll hier nicht im Detail eingegangen werden. Siehe dazu Links 2009, 19-22. schen Ägyptologie in der Mitte des 20. Jahrhunderts ist.

Das vorgestellte Dokument stammt aus einer Zeit, in der es im deutschen Sprachraum noch kaum wieder ägyptologische Publikationen gab. Deutsche Ägyptologen, so sie denn wieder publizieren wollten und konnten, mussten zumeist auf ausländische Organe ausweichen ${ }^{6}$. Da es im Ausland durchaus Vorbehalte gegen deutsche Ägyptologen gab, besonders gegenüber solchen Fachvertretern, die sich durch ihr Verhalten in der Zeit des Nationalsozialismus diskreditiert hatten, lag die Wiederbelebung der deutschen Fachorgane im Interesse gerade derjenigen Personen, für die dies zutraf. Zu diesen gehörte auch Hermann Grapow ${ }^{\top}$. Darüber hinaus waren Hermann Grapow, aber auch andere bekannte Ägyptologen, wie Hermann Kees, bereits lange mit dem Verlag verbunden und hatten so einen Einfluss auf das, was publiziert wurde ${ }^{8}$. Daher war für diese Wissenschaftler eine Aufrechterhaltung der alten Verbindung mit dem Verlag J. C. Hinrichs wünschenswert, um diesen Ein-

\footnotetext{
${ }^{6}$ Ohne eine exakte statistische Erhebung durchgeführt zu haben, drängt sich dieser Eindruck bei der Lektüre der Annual Egyptological Bibliography der Jahre 1947-1950 auf (J anssen 1948 ff.).

Auf seine Verwicklungen in der NS-Zeit kann an dieser Stelle nicht eingegangen werden. Es sei aber auf seine Erwähnung in der sog. „Steindorff-Liste“ verwiesen, die klar zeigt, dass seine Rolle im Ausland bekannt gemacht worden war. Siehe dazu Schneider 2012 und Franzmeier, Weber 2013, 133-141.

${ }^{8}$ Siehe dazu allgemein Franzmeier, Weber 2013, 116-118. Für einen Einzelfall siehe ibid., 128-133. Dort wird von Hermann Kees gegen die Publikation eines Werkes von Günther Roeder argumentiert.
} 
fluss auch im Nachkriegsdeutschland zu bewahren. Die Protagonisten waren sich dieser Situation bewusst und bewerteten vor diesem Hintergrund Neugründungen, wie pejorative Kommentare aus Briefen belegen, die ebenfalls in der Autorenkorrespondenz des Verlages J. C. Hinrichs erhalten geblieben sind ${ }^{9}$. Somit ist es kaum verwunderlich, dass Hermann Grapow nach dem Krieg dem Verlag bei den Versuchen zur Erlangung einer Drucklizenz, hier ganz spezifisch für die ZÄS, hilft.

Die Argumentationsstränge im Antrag zeigen die Strategie der Protagonisten und lassen auch Rückschlüsse auf das Selbstverständnis von Hermann Grapow im Hinblick auf die deutsche Ägyptologie im Allgemeinen zu.

An erster Stelle steht dabei der Hinweis auf die große Tradition seit dem Jahr 1863. Es folgt eine Auflistung aller ehemaligen Herausgeber. Bemerkenswert ist, dass hier keine Bewertung dieser Personen vorgenommen wird. Dies trifft sowohl auf unstrittige Größen der Ägyptologie, wie Adolf Erman oder Heinrich Brugsch zu, als auch auf Walther Wolf, den man trotz seiner Belastung durch seine Rolle im Dritten Reich neben das Opfer Georg Steindorff stellt ${ }^{10}$.

\footnotetext{
'Siehe dazu bei Franzmeier, Weber 2013, 128-133. In einem dort zitierten Brief wird ebenfalls von Hermann Kees der neugegründete Hans-PuttyVerlag als „unsympathische Neugründung“ von „Konjunkturrittern“ bezeichnet. Abschrift eines Briefes von Hermann Kees an Leopold Klotz, 22.02.1947 (Sächsisches Staatsarchiv, Staatsarchiv Leipzig, 22208 J. C. Hinrichs Verlag, Leipzig, Nr. 659, Bl. 79).

${ }^{10} \mathrm{Zu}$ Walther Wolf siehe Schneider 2012, $187-$ 210. Ungeachtet der Frage nach seiner tatsächlichen Schuld und seinem Verhalten im Dritten Reich war sein Ruf offenbar nicht gut. Wie wichtig die Freiheit von Belastung in diesem Fall war, zeigt ein bei Schneider 2012, 189 dokumentierter Sachverhalt. Der erste Nachkriegsrektor der Leipziger Universität, Hans-Georg Gadamer, beantwortet die Anfrage der Deutschen Zentralverwaltung für Volksbildung in der Sowjetischen Besatzungszone im Oktober 1946, in der es um die Bewertung Wolfs vor dem Hintergrund der Wiederaufnahme der ZÄS geht, mit der Aussage, dass Wolf noch nicht aus dem Wehrdienst zurückgekommen sei, eine Bewertung ihm daher unmöglich sei. Dies tut er vor dem Hintergrund, dass jedwede politische Belastung ein großes Problem darstellt, wie er bereits vorher an Wolf geschrieben hatte.
}

Den zentralen Baustein der Argumentation für die Lizenzerteilung der ZÄS bildet die Darstellung der wichtigen Position der Zeitschrift innerhalb der internationalen Ägyptologie. Es wird darauf verwiesen, dass die ZÄS die internationale Fachzeitschrift für das Fach ist. Alle vergleichbaren Organe werden sehr selbstbewusst als „ephemer" bezeichnet. Es wird hervorgehoben, dass die Autoren in der Vergangenheit internationaler Herkunft waren. In der Auflistung der beteiligten Nationen steht dann auch Russland nach Deutschland an zweiter Stelle, sicherlich kein Zufall. Daran schließen sich die drei westlichen Besatzungsmächte Großbritannien, USA und Frankreich an, weitere Länder folgen.

Nach dieser Bewertung der ZÄS wird darauf verwiesen, dass die Zeitschrift in den Händen des Verlages J. C. Hinrichs bleiben solle, der sich um die Ägyptologie große Verdienste erworben habe. Dieses Eintreten für den Verlag macht klar, dass es Grapow nicht allein um die Zeitschrift und die Ägyptologie ging. Vielmehr wusste er, dass er so seinen Einfluss würde behalten können, auch ohne persönlich offen in Erscheinung treten zu müssen ${ }^{11}$.

Diesen Versuch der Einflussnahme setzt er auch in seinem Vorschlag für die Besetzung des Postens des Herausgebers fort. Er schlägt mit dem noch sehr jungen Fritz Hintze (1915-1993) einen eigenen Schüler und Mitarbeiter vor ${ }^{12}$. Es kann davon ausgegangen werden, dass sich Grapow davon einen fortdauernd guten Kontakt zu Zeitschrift und Verlag versprach. In der sowjetisch besetzten Zone hätten jedoch kaum andere Personen zur Verfügung gestanden ${ }^{13}$. Alle älte-

${ }^{11}$ Hermann Grapow pflegte ein enges, fast freundschaftlich zu nennendes Verhältnis zu Leopold Klotz, dem Geschäftsführer des Verlages J. C. Hinrichs, und konnte auf diesem Weg Einfluss nehmen. So wurde Grapow von Klotz mehrfach um Hilfe ersucht, wenn es um die Bewertung eingereichter Manuskripte ging. Siehe für einen konkreten Fall Franzmeier, Weber 2013, 133-141.

${ }_{12}$ Siehe zu Fritz Hintze Endesfelder 1988, 5664 und Krauspe, Hornung, Blumenthal 1993. Es ist an dieser Stelle klar hervorzuheben, dass sich Fritz Hintze letztlich als fähiger Herausgeber erwies, der bis zu seinem Tod 1993 in der Position verblieb.

${ }_{13}$ Der einzige andere Ägyptologe auf dem Gebiet der ehemaligen DDR, der infrage kam, war der ein Jahr 
ren und renommierten Ägyptologen, auch solche wie Alexander Scharff, Hans Bonnet oder Rudolf Anthes, der mehrfach, etwa von Georg Steindorff, ins Gespräch gebracht worden war, lebten in den westlichen Besatzungszonen, so wie auch Hermann Grapow selbst ${ }^{14}$. Ein Wohnsitz des zukünftigen Herausgebers in der sowjetischen Besatzungszone war jedoch unabdingbare Voraussetzung für die Bewilligung der Lizenz, wie explizit aus dem Antrag hervorgeht.

Das letzte vorgebrachte Argument ist ein möglicher Gang in den Westen. Dies ist sicherlich vor dem Hintergrund der bereits erwähnten, gleichzeitigen Verlagsgründungen im Westen Deutschlands zu sehen. Wäre von Kollegen im Westen eine neue Zeitschrift mit dem Anspruch gegründet worden, die Stellung der ZÄS zu übernehmen, so wären die alten Strukturen, in denen Grapow eine solch zentrale Stellung hatte, vermutlich völlig zerstört worden.

Letztlich kann konstatiert werden, dass Grapows Strategie aufging, auch wenn dies noch einige Jahre dauern sollte. Im Jahr 1954 erschien die ZÄS tatsächlich mit den Herausgebern Siegfried Morenz und Fritz Hintze im Ost-Berliner Akademie-Verlag ${ }^{15}$, zunächst noch in Arbeitsgemeinschaft mit dem Verlag J. C. Hinrichs.

\section{Literatur}

Endesfelder, E., 1988, Die Ägyptologie an der Berliner Universität - Zur Geschichte eines Fachgebietes, Berlin.

Franzmeier, H., Weber, A., 2013, „Andererseits finde ich, dass man jetzt nicht so tun soll, als wäre nichts gewesen." Die deutsche Ägyptologie in den Jahren 1945-1949 im Spiegel der Korrespondenz mit dem Verlag J. C. Hinrichs, in: S. Bickel, H.-W. Fischer-Elfert, A. Loprie- no, S. Richter (Hsgg.), Ägyptologen und Ägyptologien zwischen Kaiserreich und Gründung der beiden deutschen Staaten. Reflexionen zur Geschichte und Episteme eines altertumswissenschaftlichen Fachs im 150. Jahr der Zeitschrift für Ägyptische Sprache und Altertumskunde Berlin, 113-152.

Geist, L., 1991, Ein Geschäft recht geistiger Natur. Der J. C. Hinrichs Verlag, Leipzig.

Janssen, J. M. A., 1948 ff., Annual Egyptological Bibliography, Leiden.

Krauspe, R., Hornung, E., Blumenthal, E., 1993, „Fritz Hintze. 18. April 1915-30. März 1993“, ZÄS 120, IX-XI.

Links, C., 2009, Das Schicksal der DDR-Verlage. Die Privatisierung und ihre Konsequenzen, Berlin.

Schneider, T., 2012, Ägyptologen im Dritten Reich. Biographische Notizen anhand der sogenannten „Steindorff-Liste“, in: T. Schneider, P. Raulwing (Hgg.), Egyptology from the First World War to the Third Reich, Leiden, 120-247.

\section{Summary}

Between the years 1944 and 1954, the German Egyptological journal Zeitschrift für Ägyptische Sprache und Altertumskunde (ZÄS) stopped publication due to the Second World War and its repercussions. This paper presents a document written by Hermann Grapow in 1947 intended to help the publishing house J. C. Hinrichs obtain a licence from the authorities in the Soviet Occupation Zone, in order to publish the ZÄS anew. Here, emphasis will be placed upon the way in which Hermann Grapow argues in favour of the grant of the licence in his petition.

\section{Keywords}

Egyptology - Grapow, Hermann - J. C. Hinrichs postwar period - ZÄS ältere Siegfried Morenz in Leipzig, der schließlich 1954 zusammen mit Fritz Hintze Herausgeber der wiederbegründeten ZÄS wurde.

${ }^{14}$ Hermann Grapow lebte bis $\mathrm{zu}$ seinem Tod in West-Berlin. Siehe Endes felder 1988, 54.

${ }^{15}$ Zum Akademie-Verlag siehe zuletzt überblickend Links 2009, 49-52. 
TAFEL XVII

\author{
Antrag auf lizenzerteilung fix die \\ "Zeitschxift fur ägyptische Sprache und Altertumskunde" \\ Herauggegeben in Verbindung mit anderen Fachgelehrten
}

von Drophil. Fritz Hintze.

Verlag: J.C.Hinrichs/Leopold Klotz Leipzig/Gotha.

Die Zeitschrift fir ägyptische Sprache und Altertumskunde ist 1863 von Heinrich Brugsch-Pascha begrindet worden. Sie 1st seitdem Jahr un Jahr in ununterbrochenex Folge bis zum Jahre 1944 erschienen, in dem ihr 78. Bend auggegoben porden ist. Die friheren Herausgeber waren Heinrich Brugsch (er fuhrte die Reaktion bis zu seinem Tode im Jahre 1894 teils allein, teils in Verbindung mit Richard Lepsius und Adolf Erman), Adolf Jrman (er leitete die Zeitsohrift teils allein, teile in Verbindung mit Georg Steinforef bis etwa 1920), Georg Steindoref (er gab die Zeitschrift heraus bis zum Jahre 1938, in den letzten Jahren zusammen mit Walthex Wolf), Walther Wolf seit 1938. Die Zeitschrift ist im Besitz dex oben genannten Verlagsbuchhandlung, die bislang bei den erwâhnten Wechseln in der Ferausgabe jedesmal ein entscheidendes Wort mitzusprechen natte.

Die Zeitschrift eir ëgyptische Sprache und Altertumskunde ist $d i e$ ägyptologische Fachzeitschrift fir In- und Assland von unbestrittenem Ansehen und in ungeminderter Bedeutung seit ihrem Bestehen gewesen. wit Befträgen von deutschen, russischen, englischen, anerikanischen, französischen, dänischen, italienischen, schweizerischen, ägyptischen, schwedischen, norwegtschen, tschechischen, österreichischen, holländischen, belgischen Ägyptologen. Die ÄgYptologie hat diese Zeitschrift immer als thr eigentilches zentralorgan angesehen, unbeschadet dex Pachzeitschriften, die es rehr oder weniger ephemer in einer Rejhe von Jindern daneben gegeben hat oder gibt.

Diese ägyptologische zeitschrift von wahrhaft internationaler Bigenart, die in ihren Aufsätzen die genze Geschichte der Ägyptologie von rund 80 Jahren wiederspiegelt, sollte unter allen Umständen am Leben erhalten das heibt fortgefihrt werden. Sie sollte debei

Antrag auf Lizenzerteilung (zu Franzmeier und Weber, Lizenzerteilung). 
im Besitz der oben genannten Veriagsbuchhandlung bleiben, die sich wie un die $\overparen{R g y t o l o g i e}$ uberheupt so insbesondere um die "Zeitschrift" die grobten Verdienste exworben hat.

Alg kinitiger lereuggeber kormt für den russischen Beatzungsbereich - soll anders nicht ein kgytologe aus einer der ubrigen Besatzungszonen narhaft gemacht werden, was doch vermieden werden muß Dr.phil. Fritz Hintze,Potsdam, Sanrlandanger 11, in Betracht. Dr.Hintze 1st wissenschefticher kitarbeiter der Deutschen Akaderie lìer Wissenschaften beim wrterbuch der R̈Eptischen Sprache (Ägyptologische Kommission, kunftig im Rehren des besntragten Instituts fü Erforschung des Alten Orients) und steht in Fegrife, sich in der philosophischen Rakultitt der Universitat Berlin fur des Fach der Rgyptologie zu habilitieren. Dr. Hintze ist trotz seiner relativen Jugend (Anfang der DreiBiger) wissenschaftich und personllch durchaus fitr die Leitung der Zeitschrift geelgnet. Br wirde darüber hinaus zugleich die sehr winschenswerte Verbindung mit dex Universitat vermitteln.

Im Hinblick derauf, das zweifellos Bestrebungen im Gange sind, wie so viele andere wisgenschaftliche organe auch diese Zeitschrift aus der sowjetischen Besatzungezone in eine der anderen zonen hertherzuziehen, wäre eine baldige gitnstige Entscheldung sehr erwïnscht.

Berlin, den 27.Januar 1947

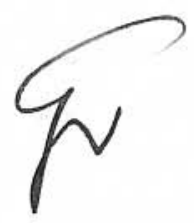

Antrag auf Lizenzerteilung (zu Franzmeier und Weber, Lizenzerteilung). 